Pacific Journal of Mathematic 


\title{
INJECTIVE MODULES OVER NOETHERIAN RINGS
}

\author{
EBEN MATLIS
}

Introduction In this discussion every module over a ring $R$ will be understood to be a left $R$-module. $R$ will always have a unit, and every module will be unitary. The aim of this paper is to study the structure and properties of injective modules, particularly over Noetherian rings. B. Eckmann and A. Schopf have shown that if $M$ is a module over any ring, then there exists a unique, minimal, injective module $E(M)$ containing it. The module $E(M)$ will be a major tool in our investigations, and we shall systematically exploit its properties.

In $\S 1$ we show that if a module $M$ has a maximal, injective submodule $C$ (as is the case for left-Noetherian rings), then $C$ contains a carbon-copy of every injective submodule of $M$, and $M / C$ has no injective submodules different from 0 . Although $C$ is unique up to an automorphism of $M, C$ does not in general contain every injective submodule of $M$. In fact, the sum of two injective submodules of a module is always injective if and only if the ring is left-hereditary.

In $\S 2$ we show that for any ring $R$ a module $E$ is an indecomposable, injective module if and only if $E \cong E(R / J)$, where $J$ is an irreducible, left ideal of $R$. We prove that if $R$ is a left-Noetherian ring, then every injective $R$-module has a decomposition as a direct sum of indecomposable, injective submodules. Strong uniqueness assertions can be made concerning such decompositions over any ring.

In $\S 3$ we take $R$ to be a commutative, Noetherian ring, and $P$ to be a prime ideal of $R$. We prove there is a one-to-one correspondence between the prime ideals of $R$ and the indecomposable, injective $R$ modules given by $P \leftrightarrow E(R / P)$. We examine the structure of the module $E=E(R / P)$, and show that if $A_{i}$ is the annihilator in $E$ of $P^{i}$, then $E=\cup A_{i}$ and $A_{i+1} / A_{i}$ is a finite dimensional vector space over the quotient field of $R / P$. The ring of $R$-endomorphisms of $E$ is isomorphic in a natural way to $\bar{R}_{p}$, the completion of the ring of quotients of $R$ with respect to $R-P$. As an $\bar{R}_{p}$-module $E$ is an injective envelope of $\bar{R}_{p} / \bar{P}$, where $\bar{P}$ is the maximal ideal of $\bar{R}_{p}$. If $P$ is a maximal ideal of $R$, then $E$ is a countably generated $R$-module. Every indecomposable, injective $R$-module is finitely generated if and only if $R$ has the minimum condition on ideals.

In $\S 4$ we take $R$ to be a commutative, Noetherian, complete, local ring, $P$ the maximal ideal of $R$ and $E=E(R / P)$. Then the contravariant,

Received April 10, 1958. In revised form June 9, 1958. 
exact functor $\operatorname{Hom}_{R}(A, E)$ establishes a one-to-one correspondence between the category of modules with ACC and the category of modules with DCC such that $\operatorname{Hom}_{R}\left(\operatorname{Hom}_{R}(A, E), E\right) \cong A$, for $A$ in either category. In particular, there is a lattice anti-isomorphism of the ideals of $R$ and the submodules of $E$ given by annihilation.

This paper is essentially the first half of a doctoral dissertation submitted to the University of Chicago. The author would like to express his deep appreciation for the assistance and inspiration given to to him by Professor I. Kaplansky.

\section{Maximal Injective Submodules.}

Definition Let $R$ be a ring and $S$ an $R$-module. Then an $R$-module $M$ is said to be an essential extension of $S$, if $S \subset M$, and if the only submodule $T$ of $M$ such that $S \cap T=0$ is the submodule $T=0$.

B. Eckmann and A. Schopf have shown [4] that if $R$ is a ring and $M$ an $R$-module, then there exists an injective $R$-module $E(M)$ which is an essential extension of $M$. If $C$ is any other injective extension of $M$, then there exists in $C$ a maximal, essential extension $A$ of $M$, and there is an isomorphism of $E(M)$ onto $A$ which is the identity on $M$. We shall call $E(M)$ the injective envelope of $M$. A. Rosenberg and D. Zelinsky have called this module the injective hull of $M$ [9].

Lemma 1.1. Let $R$ be a ring, $S$ and $T R$-modules, and $D$ an injective submodule of $S \oplus T$. Let $E$ be an injective envelope of $D \cap S$ in $D$, and let $F$ be a complementary summand of $E$ in $D$. Thus $D=E \oplus F$; and $E$ and $F$ project monomorphically into $S$ and $T$, respectively.

Proof. It is clear that $F$ projects monomorphically into $T$. Let $f$ be the projection of $E$ into $S$. Since $\operatorname{Ker} f \subset T, \operatorname{Ker} f \cap(D \cap S)=0$. However, $E$ is an essential extension of $D \cap S$, and so $\operatorname{Ker} f=0$.

The following proposition is well known [3, Ch. 1, Ex. 8], but we state it for the sake of reference.

Proposition 1.2. Let $R$ be a left-Noetherian ring. Then:

(1) A direct limit of injective $R$-modules is injective.

(2) $A$ direct sum of $R$-modules is injective if and only if each summand is injective.

It is an immediate consequence of Proposition 1.2 that if $R$ is a left-Noetherian ring and $M$ an $R$-module, then $M$ possesses a maximal, injective submodule $C$. Concerning this situation we have the following:

Theorem 1.3. Let $R$ be any ring and $M$ an $R$-module such that $M$ has a maximal, injective submodule $C$. Then: 
(1) If $N$ is a complementary summand of $C$ in $M$, then $M=C \oplus N$, and $M / C \cong N$ has no injective submodules different from 0 .

(2) If $E$ is any injective submodule of $M$, then the projection of $E$ into $C$ maps $E$ isomorphically onto an injective envelope of $E \cap C$ in $C$.

(3) If $D$ is any other maximal, injective submodule of $M$, then there is an automorphism of $M$ which carries $C$ onto $D$ and is the identity on $N$.

Proof. (1) is obvious, and (2) follows immediately from (1) and Lemma 1.1. We will prove (3). Let $f$ be the projection of $D$ into $C$. By (2) $f(D)$ is an injective envelope of $D \cap C$ in $C$; and thus $C=$ $f(D) \oplus C_{1}$, where $C_{1}$ is an injective submodule of $C$. However, since $(D \cap C) \subset f(D), D \cap C_{1}=0$; and thus by the maximality of $D, C_{1}=0$. Therefore, since $f$ is one-to-one by (2), $f$ is an isomorphism of $D$ onto $C$, and so $D \cap N=0$. Thus $M=D \oplus N$, and this completes the proof of the theorem.

If the sum of the injective submodules of a module $M$ is always injective, then $M$ has a unique, maximal, injective submodule which contains every injective submodule of $M$. However, in general, this is not the case. In fact, we have the following.

TheOREm 1.4. Let $R$ be any ring. The sum of two injective submodules of an $R$-module is always injective if and only if $R$ is lefthereditary.

Proof. If $R$ is left-hereditary and $N_{1}, N_{2}$ are injective submodules of an $R$-module $N$, then $N_{1}+N_{2}$ is a homomorphic image of the injective $R$-module $N_{1} \oplus N_{2}$, and hence is injective.

Conversely, assume that the sum of two injective submodules of any $R$-module is injective. Let $M$ be any injective $R$-module and $H$ a submodule of $M$. We will show that $M / H$ is injective, and this will prove that $R$ is left-hereditary.

Let $M_{1}, M_{2}$ be two copies of $M, N=M_{1} \oplus M_{2}$, and $D$ the submodule of $N$ consisting of the elements $(h, h)$, where $h \in H$. The canonical homomorphism : $N \rightarrow N / D$ maps $M_{1}, M_{2}$ isomorphically onto submodules $\bar{M}_{1}, \bar{M}_{2}$ of $N / D$, respectively. Since $N / D=\bar{M}_{1}+\bar{M}_{2}, N / D$ is injective ; and, therefore, $(N / D) / \bar{M}_{1}$ is injective. The composite mapping: $M \rightarrow$ $M_{2} \rightarrow \bar{M}_{2} \rightarrow(N / D) / \bar{M}_{1}$ defines a homomorphism of $M$ onto $(N / D) / \bar{M}_{1}$ with kernel $H$. Therefore, $M / H$ is injective; and the proof is complete.

It follows easily from Proposition 1.2 and Theorem 1.4 that if $M$ is any module over a left-Noetherian, left-hereditary ring, then $M$ has a unique, maximal, injective submodule $C$ which contains every injective submodule of $M$. 


\section{Indecomposable Injective Modules.}

Proposition 2.1. Let $R$ be a ring and $\left\{M_{i}\right\}$ a finite family of $R$ modules. Then the natural imbedding of $\sum \oplus M_{i}$ into $\Sigma \oplus E\left(M_{i}\right)$ can be extended to an isomorphism of $E\left(\sum \oplus M_{i}\right)$ and $\sum \oplus E\left(M_{i}\right)$. If $R$ is left-Noetherian, the finiteness restriction can be omitted.

Proof. We identify $\Sigma \oplus M_{i}$ with its image in $\Sigma \oplus E\left(M_{i}\right)$. If the family is finite, or if $R$ is left-Noetherian, the latter module is injective. Hence it suffices to show that $\sum \oplus E\left(M_{i}\right)$ is an essential extension of $\Sigma \oplus M_{i}$, regardless of whether the family is finite or not. Let $x \neq 0 \in \Sigma \oplus E\left(M_{i}\right)$; then since $x$ has only a finite number of non-zero coordinates, we can by working successively on each coordinate find an element $r \in R$ such that $r x \neq 0$ and $r x \in \sum \oplus M_{i}$. This proves the assertion.

Definition. Let $A$ be a module over a ring $R$. We say that $A$ is indecomposable, if its only direct summands are 0 and $A$.

The following proposition follows readily from the definitions :

Proposition 2.2. Let $M$ be a module over a ring $R$. Then the following statements are equivalent:

(1) $E(M)$ is an injective envelope of every one of its non-zero submodules.

(2) $M$ contains no non-zero submodules $S$ and $T$ such that $S \cap T=0$.

(3) $E(M)$ is indecomposable.

In general it is not true that if $M$ is indecomposable, then $E(M)$ is also indecomposable. For let $M$ be an indecomposable, torsion-free module of rank two over an integral domain $R$. Let $Q$ be the quotient field of $R$. Then $E(M) \cong Q \oplus Q$ [5, Th. 19].

Definition. Let $R$ be a ring and $I$ a left ideal of $R$ such that $I=J_{1} \cap \cdots \cap J_{n}$, where $J_{i}$ is a left ideal of $R$. We call this a decomposition of $I$, and we say the decomposition is irredundant, if no $J_{i}$ contains the intersection of the others.

THEOREM 2.3. Let $R$ be a ring and $I=J_{1} \cap \cdots \cap J_{n}$ an irredundant decomposition of the left ideal $I$ by left ideals $J_{i}$. Assume that each $E\left(R / J_{i}\right)$ is indecomposable. Then the natural imbedding of $R / I$ into $C=$ $E\left(R / J_{1}\right) \oplus \cdots \oplus E\left(R / J_{n}\right)$ can be extended to an isomorphism of $E(R / I)$ onto $C$.

Proof. We identify $R / I$ with the submodule of $C$ consisting of all elements of the form $\left(r+J_{1}, \cdots, r+J_{n}\right)$, where $r \in R$. Since $C$ is 
injective, it is sufficient to show that it is an essential extension of $R / I$. We first show that $R / I \cap R / J_{i} \neq 0$ for all $i$; for simplicity we will prove only the case $i=1$. By the irredundancy of the decomposition of $I$ we can find $r \in R$ such that $r \in J_{2} \cap \cdots \cap J_{n}$, but $r \notin J_{1}$. Then $\left(r+J_{1}, r+\right.$ $\left.J_{2}, \cdots, r+J_{n}\right)=\left(r+J_{1}, 0, \cdots, 0\right)$ is a non-zero element of $R / I \cap R / J_{1}$.

By Proposition $2.2 E\left(R / J_{i}\right)$ is an injective envelope of $R / I \cap R / J_{i}$. Let $x \neq 0 \in C$. By working successively in each component we can find $s \in R$ such that $s x \neq 0$, and the $i$ th component of $s x$ is in $R / I \cap R / J_{i}$. Thus $s x$ is a sum of elements of $R / I$, and hence $s x \in R / I$. Thus $C$ is an essential extension of $R / I$.

Definition. Let $J$ be a left ideal of a ring $R$. We say that $J$ is irreducible, if there do not exist left ideals $K$ and $L$ of $R$, properly containing $J$, such that $K \cap L=J$.

Notation. Let $M$ be a module over a ring $R$, and let $S$ be a subset of $M$. Then we define $O(S)=\{r \in R \mid r S=0\}$. Clearly $O(S)$ is a left ideal of $R$.

THEOREM 2.4. A module $E$ over a ring $R$ is an indecomposable, injective module if and only if $E \cong E(R / J)$, where $J$ is an irreducible left ideal of $R$. In this case, for every $x \neq O \in E, O(x)$ is an irreducible left ideal and $E \cong E(R / O(x))$.

Proof. Let $J$ be an irreducible left ideal of $R$, and $K, L$ left ideals of $R$ such that $K / J \cap L / J=0$. Then $K \cap L=J$, and thus either $K=J$ or $L=J$. Hence $E(R / J)$ is indecomposable by Proposition 2.2.

Conversely, let $E$ be an indecomposable, injective module, $x \neq 0 \in E$, and $J=O(x)$. By Proposition $2.2 E=E(R x)$; and since $R x \cong R / J$, we have $E \cong E(R / J)$. Suppose that $J=K \cap L$ is an irredundant decomposition of $J$ by the left ideals $K, L$. We imbed $R / J$ in $C=E(R / K) \oplus E(R / L)$, and let $D$ be an injective envelope of $R / J$ in $C$. Due to the irredundancy of the decomposition of $J, R / J \cap R / K \neq 0$. Therefore, by Lemma 1.1 and the indecomposability of $D, D$ projects monomorphically into $E(R / K)$. The image of $D$ is an injective module containing $R / K$, and hence is equal to $E(R / K)$. Thus $E(R / K)$ is indecomposable; similarly, $E(R / L)$ is indecomposable. Thus by Theorem $2.3 E(R / J) \cong E(R / K) \oplus E(R / L)$. This contradicts the indecomposability of $E(R / J)$, and thus $J$ is irreducible.

\section{REMARKS.}

(1) Every ring $R$ possesses indecomposable, injective modules. For if $J$ is a maximal, left ideal of $R$ (such exist by Zorn's lemma and the fact that $R$ has an identity element), then $J$ is an irreducible ideal, 
and so $E(R / J)$ is indecomposable by Theorem 2.4 .

(2) It can be shown that every injective module over a ring $R$ has an indecomposable, direct summand if and only if every left ideal of $R$ has an irredundant decomposition by left ideals, at least one of which is irreducible.

Theorem 2.5. Let $R$ be a left-Noetherian ring. Then every injective $R$-module has a decomposition as a direct sum of indecomposable, injective submodules.

Proof. Let $M$ be an injective $R$-module. Then by Zorn's lemma we can find a submodule $C$ of $M$ which is maximal with respect to the property of being a direct sum of indecomposable, injective submodules. Suppose that $C \neq M$. By Proposition $1.2 C$ is injective, and hence there is a non-zero submodule $D$ of $M$ such that $M=C \oplus D$. Let $x \neq 0 \in D$; since $R$ is left-Noetherian, $O(x)$ is an intersection of a finite number of irreducible, left ideals [8, Lemma 1.8.2]. Therefore, by Theorems 2.3 and 2.4, $E(R / O(x))$ is a direct sum of a finite number of indecomposable, injective $R$-modules. Now $R x \cong R / O(x)$, and so we can consider that $E(R / O(x))$ is imbedded in $D$. But then $C \oplus E(R / O(x))$ contradicts the maximality of $C$, and thus $C=M$. This concludes the proof of the theorem.

Theorem 2.5 is a generalization of a theorem of Y. Kawada, K. Morita, and H. Tachikawa [6, Th. 3.2] who considered the case of a ring with the minimum condition on left ideals. Their theorem, in turn, is a generalization of a theorem of H. Nagao and T. Nakayama [7] concerning finite dimensional algebras over a field.

Definition. Following the most general definition [3, p. 147] we will call a ring $H$ a local ring if the set of non-units of $H$ forms a twosided ideal.

Proposition 2.6. Let $E$ be an injective module over $a$ ring $R$ and $H=\operatorname{Hom}_{R}(E, E)$. Then $H$ is a local ring if and only if $E$ is indecomposable. In this case $f \in H$ is a unit if and only if $\operatorname{Ker} f=0$.

Proof. If $E$ is not indecomposable, then $H$ has a non-zero idempotent different from the identity, which can't happen in a local ring. Conversely, assume that $E$ is indecomposable. If $f \in H$ is a unit, then of course $\operatorname{Ker} f=0$; while if $\operatorname{Ker} f=0$, then $f(E)$ is injective, $f(E)=E$, and so $f$ is a unit of $H$. Let $g, h$ be non-units of $H$. Then Ker $g \neq 0$, Ker $h \neq 0$, and by Proposition 2.2 Ker $g \cap$ Ker $h \neq 0$. Since Ker $g \cap \operatorname{Ker} h \subset \operatorname{Ker}(g+h)$, it follows that $g+h$ is a non-unit. It is well- 
known that if the sum of two non-units of a ring is always a non-unit, then the ring is a local ring.

The preceding proposition shows that G. Azumaya's generalization of the Krull-Remak-Schmidt theorem [1, Th. 1] applies to direct sums of indecomposable, injective modules. We state this theorem without proof in the form in which it applies to our considerations.

Proposition 2.7. Let $R$ be $a$ ring and $M=\sum \oplus E_{a}(a \in A)$ an $R$ module which is a direct sum of indecomposable, injective submodules $E_{a}$. Then:

(1) Given a second decomposition of $M$ into indecomposable submodules $F_{b}(b \in B): M=\sum \oplus F_{b}$, then there exists a one-to-one mapping $a \rightarrow b(a)$ of $A$ onto $B$ such that $E_{a}$ is isomorphic to $F_{b(a)}$, for each $a \in A$. In other words the decomposition of $M$ into indecomposable submodules is unique up to an automorphism of $M$.

(2) For any non-zero idempotent element $f$ in $\operatorname{Hom}_{R}(M, M)$ there exists at least one $E_{a}$ such that $f$ is an isomorphism on $E_{a}$. In particular every indecomposable, direct summand of $M$ is isomorphic to one of the $E_{a}^{\prime}$ s.

It is an open question whether every direct summand of $M$ is also a direct sum of indecomposable, injective modules. Of course if $R$ is left-Noetherian, then Theorem 2.5 provides an affirmative answer.

The following proposition will be needed in $\S 3$.

Proposition 2.8. Let $R$ be a ring, $B$ an $R$-module, and $C$ an injective $R$-module; let $y \in C$ and $x_{1}, \cdots, x_{n} \in B$. Then $\cap O\left(x_{i}\right) \subset O(y)$ if and only if there exist $f_{1}, \cdots, f_{n} \in \operatorname{Hom}_{R}(B, C)$ such that $y=f_{1} x_{1}+\cdots+f_{n} x_{n}$.

Proof. If $y=f_{1} x_{1}+\cdots+f_{n} x_{n}$, then clearly $\cap O\left(x_{i}\right) \subset O(y)$. Now assume that $\cap O\left(x_{i}\right) \subset O(y) . \quad R / \cap O\left(x_{i}\right)$ is a cyclic $R$-module generated by an element $z$ with $O(z)=\cap O\left(x_{i}\right)$. There exists an $R$-homomorphism : $R z \rightarrow R y$ such that $z \rightarrow y$; and there exists a natural imbedding of $R z$ into $\sum \oplus R x_{i}$ such that $z \rightarrow\left(x_{1}, \cdots, x_{n}\right)$. This yields the diagram:

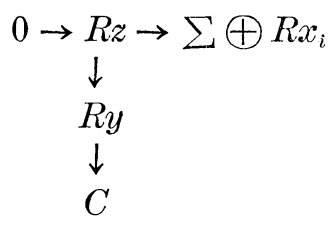

Since $C$ is injective, there is an $R$-homomorphism $f: \sum \oplus R x_{i} \rightarrow C$ such that $f\left(x_{1}, \cdots, x_{n}\right)=y$. Now $f=\sum \hat{f}_{i}$, where $\hat{f}_{i}: R x_{i} \rightarrow C$ is the restriction of $f$ to $R x_{i}$. We have the diagram: 


$$
\begin{aligned}
0 \rightarrow & R x_{i} \rightarrow B \\
\hat{f_{i} \downarrow} & \\
& C
\end{aligned}
$$

Therefore, we have $R$-homomorphisms $f_{i}: B \rightarrow C$ such that $f_{i}$ extends $\hat{f_{i}}$ for each $i$. Thus $f_{1} x_{1}+\cdots+f_{n} x_{n}=\hat{f_{1} x_{1}}+\cdots+\hat{f_{n}} x_{n}=f\left(x_{1}, \cdots, x_{n}\right)=y$.

\section{Injective Modules over a Commutative, Noetherian Ring.}

Throughout this section $R$ will be a fixed commutative, Noetherian ring.

Proposition 3.1. There is a one-to-one correspondence between the prime ideals of $R$ and the indecomposable, injective $R$-modules given by $P \leftrightarrow E(R / P)$, for $P$ a prime ideal of $R$. If $Q$ is an irreducible P-primary ideal, then $E(R / Q) \cong E(R / P)$.

Proof. It is obvious that a prime ideal is irreducible; and thus $E(R / P)$ is an indecomposable, injective module by Theorem 2.4. Let $P_{1}, P_{2}$ be two prime ideals of $R$ such that $E\left(R / P_{1}\right) \cong E\left(R / P_{2}\right)$. We consider that $R / P_{1}$ and $R / P_{2}$ are imbedded in $E\left(R / P_{1}\right)$. Hence by Proposition 2.2, $R / P_{1} \cap R / P_{z} \neq 0$. However, every nonzero element of $R / P_{1}$ (resp. $\left.R / P_{2}\right)$ has order ideal $P_{1}$ (resp. $P_{2}$ ). Thus $P_{1}=P_{2}$, and the mapping $P \rightarrow E(R / P)$ is one-to-one.

Let $E$ be any indecomposable, injective $R$-module. Then by Theorem 2.4 there is an irreducible ideal $Q$ of $R$ such that $E \cong E(R / Q)$. Now $Q$ is a primary ideal with a unique associated prime ideal $P$ [8, Lemma 1.8.3]. If $Q=P$, we are finished; hence assume that $Q \neq P$. Then there is a smallest integer $n>1$ such that $P^{n} \subset Q$. Take $b \in P^{n-1}$ such that $b \notin Q$, and denote the image of $b$ in $R / Q$ by $\bar{b}$. Clearly $O(\bar{b}) \supset P$; on the other hand if $a \in O(\bar{b})$, then $a b \in Q$, and so $a \in P$, showing that $O(\bar{b})=P$. Therefore, there is an element of $E(R / Q)$ with order ideal $P$, and thus $E(R / Q) \cong E(R / P)$ by Theorem 2.4. This completes the proof of the proposition.

Lemma 3.2. Let $P$ be a prime ideal of $R$ and $E=E(R / P)$. Then:

(1) $Q$ is an irreducible, P-primary ideal if and only if there is an $x \neq 0 \in E$ such that $O(x)=Q$.

(2) If $r \in R$-P, then $O(r x)=O(x)$ for all $x \in E$, and the homomorphism : $E \rightarrow E$ defined by $x \rightarrow r x$ is an automorphism of $E$.

Proof.

(1) This is an immediate consequence of Theorem 2.4 and Proposition 3.1. 
(2) Let $r \in R-P$; the map : $E \rightarrow E$ defined by $x \rightarrow r x$, for $x \in E$, has kernel $O$ by (1); therefore, it is an automorphism by Proposition 2.6. It follows that $O(x)=O(r x)$ for every $x \in E$.

Definition. Let $M$ be an injective $R$-module, and $P$ a prime ideal of $R$. Define

$$
M_{p}=\{x \in M \mid P \subset \operatorname{Rad} O(x)\}
$$

and

$$
N_{p}=\left\{x \in M_{p} \mid P \neq \operatorname{Rad} O(x)\right\} .
$$

Let $M=\sum \oplus E_{a}(a \in A)$ be a direct decomposition of $M$ by indecomposable, injective submodules $E_{a}$. By Proposition 3.1 there is associated with each $E_{a}$ a prime ideal which we shall designate by $P_{a}$. Define $H_{p}$ to be the direct sum of those $E_{a}$ 's such that $P_{a}=P$. We shall call $H_{p}$ the $P$-component of the decomposition of $M$; and we define the $P$-index of $M$ to be the cardinal number of summands $E_{a}$ in the decomposition of $H_{p}$.

\section{THEOREM 3.3.}

(1) $M_{p}$ is the direct sum of those $E_{a}$ 's such that $P_{a} \supset P$, and $N_{p}$ is the direct sum of those $E_{a}$ 's such that $P_{a} \supsetneqq P$. Thus $M_{p}$ and $N_{p}$ are submodules of $M$; and, in fact, are direct summands of $M$. In addition, the direct sums of $E_{a}$ 's just defined are independent of the decomposition of $M$.

(2) $M$ is a direct sum of the $P_{a}$-components $H_{p_{a}}$. We have $H_{p} \cong$ $M_{p} / N_{p}$; and $H_{p}$ is unique up to an automorphism of $M$. In other words $M$ is determined up to isomorphism by a collection of cardinal numbers, namely its $P$-indices.

If $P$ is a maximal ideal of $R$, then $H_{p}=M_{p}$, and thus $H_{p}$ is independent of the decomposition.

\section{Proof.}

(1) Let $x \neq 0 \in M$; then $x=x_{1}+\cdots+x_{n}$, where $x_{i} \neq O \in E_{a_{i}}$. Thus $O(x)=\cap O\left(x_{i}\right)$; and by Lemma $3.2(1) O\left(x_{i}\right)$ is $P_{a_{i}}$-primary. From this it follows that $\operatorname{Rad} O(x)=\cap P_{a_{i}}$. Hence it is clear that $x \in M_{p}$ if and only if $x$ is an element of the direct sum of those $E_{a}$ 's such that $P_{a} \supset P$. It also follows that $x \in N_{p}$ if and only if $x$ is an element of those $E_{a}$ 's such that $P_{a} \supsetneqq P$.

(2) This follows immediately from (1) and Proposition 2.7.

We now proceed to examine the structure of a typical indecomposable, injective $R$-module. 
TheOREM 3.4. Let $P$ be a prime ideal of $R, E=E(R / P)$, and $A_{i}=$ $\left\{x \in E \mid P^{i} x=0\right\}$. Then:

(1) $A_{i}$ is a submodule of $E, A_{i} \subset A_{i+1}$, and $E=\cup A_{i}$.

(2) $\cap_{x \in A_{i}} 0(x)=P^{(i)}$, where $P^{(i)}$ is the ith symbolic prime power of $P$.

(3) The non-zero elements of $A_{i+1} / A_{i}$ form the set of elements of $E / A_{i}$ having order ideal $P$.

(4) Let $K$ be the quotient field of $R / P$. Then $A_{i+1} / A_{i}$ is a vector space over $K$, and $A_{1} \cong K$.

\section{Proof.}

(1) Clearly $A_{i}$ is a submodule of $E$, and $A_{i} \subset A_{i+1}$. Let $x \neq 0 \in E$; then by Lemma $3.2(1) O(x)$ is a P-primary ideal. Thus there exists a positive integer $i$ such that $F^{i} \subset O(x)$, and so $x \in A_{i}$. Therefore, $E=\cup A_{i}$.

(2) By Lemma $3.2(1) \bigcap_{x \in A_{i}} O(x)$ is the intersection of all irreducible, $P$-primary ideals containing $F^{i}$. It is easily seen that this intersection is equal to $P^{(i)}$.

(3) Since $P A_{i+1} \subset A_{i}$, it follows from Lemma 3.2 (2) that every non-zero element of $A_{i+1} / A_{i}$ has order ideal $P$. Conversely, if $x \in E$ is an element such that $P x \subset A_{i}$, then $x \in A_{i+1}$. Therefore, every element of $E / A_{i}$ having order ideal $P$ is an element of $A_{i+1} / A_{i}$.

(4) If $r \in R$, denote its image in $R / P$ by $\bar{r}$. Similarly, if $x \in A_{i+1}$, denote its image in $A_{i+1} / A_{i}$ by $\bar{x}$. If $s \in R-P$, then by Lemma 3.2 (2) there exists a unique $y \in A_{i+1}$ such that $x=s y$. Define an operation of $K$ on $A_{i+1} / A_{i}$ by $(\bar{r} / \bar{s}) \cdot \bar{x}=\bar{r} \bar{y}$. It is easily verified that with this definition $A_{i+1} / A_{i}$ becomes a vector space over $K$.

Take $x \neq 0 \in A_{1}$. Since $A_{0}=0, A_{1}$ is a vector space over $K$; and so we can define a $K$-monomorphism $g: K \rightarrow A_{1}$ by $g(\bar{r} / \bar{s})=(\bar{r} / \bar{s}) \cdot x$, for $\bar{r} / \bar{s} \in K$. Let $z \neq 0 \in A_{1}$. Since $E$ is an essential extension of $A_{1}$, there exist $t, w \in R-P$ such that $t x=w z$. Thus $g(t / w)=z$, and $g$ is an isomorphism.

\section{REMARKS.}

(1) Let $I \subset P$ be an ideal of $R$, and $A(I)=\{x \in E \mid I x=0\}$. Then it follows from [2, Th. 17] that $A(I)$, considered as an $R / I$-module, is an injective envelope of $(R / I) /(P / I)$. Therefore, $A(I)$ is an indecomposable, injective $R / I$-module. We obtain from [8, Lemma 1] that $\operatorname{Hom}_{R}(R / I, E) \cong A(I)$; and if $J$ is an ideal of $R$ containing $I$, then $\operatorname{Hom}_{R}(J / I, E) \cong A(I) / A(J)$.

(2) If $P=0, R$ is an integral domain, and $E=A_{1} \cong K$, which is then the quotient field of $R$. If $P \neq 0$, the module $E$ is the natural generalization of the typical divisible, torsion, Abelian group $Z_{p_{\infty}}$. We 
will show in a later theorem that $A_{i+1} / A_{i}$ is a finite dimensional vector space over $K$, although in general it is not one dimensional as is the case for $Z_{p_{\infty}}$. The ring of endomorphisms of $Z_{p_{\infty}}$ is the $p$-adic integers. This will generalize to the theorem that the ring of $R$-endomorphisms of $E$ is $\bar{R}_{p}$, the completion of the generalized ring of quotients of $R$ with respect to $R-P$.

Definition. Let $P$ be $\mathrm{b}$ prime ideal of $R, S=R-P$, and $N$ the $S$-component of the 0 -ideal of $R$; i.e. $N=\{a \in R \mid$ there exists an element $c \in S$ with $c a=0\}$. Let $R^{*}=R / N, P^{*}=P / N$, and let $S^{*}$ be the image of $S$ in $R^{*}$. No element of $S^{*}$ is a zero divisor in $R^{*}$, and thus we can form the ordinary ring of quotients of $R^{*}$ with respect to $S^{*}$. We denote this ring by $R_{p}$; it is a commutative, Noetherian, local ring with maximal ideal $P^{\prime}=R_{p} P$. We will denote the completion of $R_{p}$ by $\bar{R}_{p}$, and its maximal ideal by $\bar{P}$. Further details of the construction may be found in $[8, \mathrm{Ch} .2]$.

Lemma 3.5. Let $K$ be the quotient field of $R / P$. Then $K$ and $R_{p} / P^{\prime}$ are isomorphic as fields; and $P^{i} / P^{i+1} \otimes_{R / P} K$ and $P^{i} / P^{i+1}$ are isomorphic as vector spaces over $K$.

Procf. Let $r \in R, s \in R-P$ and denote the images of $r, s$ in $R / N$ by $r^{*}, s^{*}$, and their images in $R / P$ by $\bar{r}, \bar{s}$. It is easily verified that the mapping : $K \rightarrow R_{p} / P^{\prime}$ defined by $\bar{r} / \bar{s} \rightarrow\left(r^{*} / s^{*}\right)+P^{\prime}$ is a field isomorphism. We make every $R_{p} / P^{\prime}$-module into a $K$-module by means of this isomorphism.

Let $q \in P^{i}$ and denote its image in $R / N$ by $q^{*}$. We define a mapping : $P^{i} / P^{i+1} \otimes_{R / P} K \rightarrow P^{i} / P^{i+1}$ by $\left[q+P^{i+1}\right] \otimes \bar{r} / \bar{s} \rightarrow(\bar{r} / \bar{s})\left[q^{*}+P^{\prime i+1}\right]$. We define a mapping in the reverse direction by $q^{*} / s^{*}+P^{i+1} \rightarrow\left[q+P^{i+1}\right] \otimes \bar{r} / \bar{s}$. These mappings are $K$-homomorphisms, and their product in either order is the identity mapping. Thus $P^{i} / P^{i+1} \otimes_{R / P} K$ is isomorphic as a vector space over $K$ to $P^{i} / P^{i+1}$.

It is easily seen that if $Q$ is a $P$-primary ideal, then $N \subset Q$. Hence by Lemma $3.2(1) E(R / P)$ is an $R^{*}$-module. We will henceforth assume (without loss of generality) that $N=0$. The simplification amounts to this : $R_{p}$ is an ordinary ring of quotients of $R$.

THEOREM 3.6. With the notation as already given let $E=E(R / P)$. Then $E$ is in a natural way an $\bar{R}_{p}$-module, and as such it is an injective envelope of $\bar{R}_{p} / P$. The $R$-submodules $A_{i}=\left\{x \in E \mid P^{i} x=0\right\}$ of $E$ are equal to the corresponding submodules defined for $\bar{R}_{p}$. 
Proof. We define on operation of $R_{p}$ on $E$ as follows: let $x \in E$, $r \in R$, and $s \in R-P$; then by Lemma 3.2 (2) there exists a unique $y \in E$ such that $x=s y$. Define $(r / s) x=r y$. It is easily verified that with this definition $E$ becomes an $R_{p}$-module. If $G$ is an $R_{p}$-module containing $E$, then $E$ is an $R$-direct summand of $G$. But then $E$ is an $R_{p}$-direct summand of $G$ also, and thus $E$ is $R_{p}$-injective. Since $E$ is indecomposable as an $R$-module, it is a fortiori indecomposable as an $R_{p}$-module.

If $x \in E$ has order ideal $O(x)$ in $R$, then a straightforward calculation shows that the order ideal of $x$ in $R_{p}$ is $R_{p} O(x)$. From this it follows readily that $P^{i} x=0$ if and only if $P^{\prime i} x=0$. These remarks show that we may assume that $R$ is a local ring with maximal ideal $P$. We denote its completion by $\bar{R}$, and the maximal ideal of $\bar{R}$ by $\bar{P}=\bar{R} P$.

Let $a \in \bar{R}$; then there exists a Cauchy sequence $\left\{a_{n}\right\}$ in $R$ such that $a_{n} \rightarrow a$. Let $x \in E$; then $x \in A_{i}$ for some $i$. There exists an integer $N$ such that $a_{n}-a_{m} \in P^{i}$ whenever $n>m \geqq N$. Thus $a_{n} x=a_{m} x$, and if we define $a x=a_{N} x$, it is easily verified that this definition makes $E$ into an $\bar{R}$-module.

Let $\bar{E}$ be the $\bar{R}$-injective envelope of $E$. Since $A_{1} \cong R / P \cong \bar{R} / \bar{P}$ over $\bar{R}$, it follows from Proposition 2.2 that $\bar{E}$ is the $\bar{R}$-injective envelope of $\bar{R} / \bar{P}$. Therefore, by Theorem $3.4 \bar{E}=\cup \overline{A_{i}}$, where $\bar{A}_{i}=\left\{x \in \bar{E} \mid \overline{P^{i}} x=0\right\}$. As an $R$-module $\bar{E}$ splits into a direct sum of $E$ and an $R$-module C. If $x \in C$, then $x \in \bar{A}_{i}$ for some $i$; and if $r \in \bar{R}$, there exists $r \in R$ such that $\bar{r} \equiv \bar{r}\left(\bmod \bar{P}^{i}\right)$. Hence $\bar{r} x=r x \in C$, and thus $C$ is an $\bar{R}$ module. However, $\bar{E}$ is indecomposable as an $\bar{R}$-module; and thus $C=0$ and $\bar{E}=E$. A simple calculation shows that if $x \in E$ has order ideal $O(x)$ in $R$, then it has order ideal $\bar{R} O(x)$ in $\bar{R}$ From this it follows readily that $\bar{A}_{i}=A_{i}$.

THEOREM 3.7. With the notation of Theorem 3.6 let $H=\operatorname{Hom}_{R}(E, E)$. Then $H$ is $R$-isomorphic to $\bar{R}_{p}$; more precisely, every R-homomorphism of $E$ into itself can be realized by multiplication by exactly one element of $\bar{R}_{p}$.

Proof. It is easily seen that $H=\operatorname{Hom}_{\bar{R}_{p}}(E, E)$. Therefore, by Theorem 3.6 we can assume without loss of generality that $R$ is a complete, local ring with maximal ideal $P$. Since $\cap P^{i}=0$, if $r \neq 0 \in R$, there exists an integer $i$ such that $r \notin P^{i}$. Hence $r A_{i} \neq 0$ by Theorem 3.4 (2); and $E$ is a faithful $R$-module. Consequently, we can identify $R$ with the subring of $H$ consisting of multiplications by elements of $R$.

Define $H_{i}=\left\{h \in H \mid h\left(A_{i}\right)=0\right\}$. Then $\cap H_{i}=0$, and since $g\left(A_{i}\right) \subset A_{i}$ 
for every $g \in H, H_{i}$ is a two-sided ideal of $H$. We will prove by induction on $i$ that if $f \in H$, then for each integer $i$ there exists an element $p_{i} \in R$ such that $f \equiv p_{i}\left(\bmod H_{i}\right)$. Since $A_{1} \cong R / P$, there exists $y \in A_{1}$ such that $A_{1}=R y$. Let $f \in H$; then there exists $p_{1} \in R$ such that $f y=p_{1} y$. Thus $f \equiv p_{1}\left(\bmod H_{1}\right)$. We assume the assertion has been proved for $i \geqq 1$.

Let $P^{i+1}=Q_{1} \cap \cdots \cap Q_{n}$ be an irredundant decomposition of $P^{i+1}$ by irreducible, $P$-primary ideals $Q_{j}$. By Lemma $3.2(1)$ we can find elements $x_{j} \in A_{i+1}$ such that $O\left(x_{j}\right)=Q_{j}$ for $j=i, \cdots, n$. By the irredundancy of the decomposition we can find $q_{j} \in Q_{1} \cap \cdots \cap \hat{Q}_{j} \cap \cdots Q_{n}$ (where $\hat{Q}_{j}$ means $Q_{j}$ is left out) such that $q_{j} \in Q_{j}$, Thus $q_{j} x_{j} \neq 0$ and $q_{j} x_{k}=0$ for $j \neq k$.

By induction there exists $p_{i} \in R$ such that $g=f-p_{i} \in H_{i}$. Since $P x_{j} \subset A_{i}, \quad P\left(g x_{j}\right)=g\left(P x_{j}\right)=0$; and thus $g x_{j} \in A_{1}$. Therefore, by Proposition 2.2 there exists $r_{j} \in R$ such that $r_{j}\left(q_{j} x_{j}\right)=g x_{j}$. Let $q=r_{1} q_{1}+\cdots+r_{n} q_{n}$. Then $g x_{j}=q x_{j}$ for $j=1, \cdots, n$.

Let $x \in A_{j+1}$; then $O(x) \supset P^{i+1}=O\left(x_{1}\right) \cap \cdots \cap O\left(x_{n}\right)$. Hence by Proposition 2.8 there exist $f_{1}, \cdots, f_{n} \in H$ such that $x=f_{1} x_{1}+\cdots+f_{n} x_{n}$. By the induction hypothesis $f_{j}=s_{j}+h_{j}$, where $s_{j} \in R$ and $h_{j} \in H_{i}$. Since

$$
P x_{j} \subset A_{i}, P\left(h_{j} x_{j}\right)=h_{j}\left(P x_{j}\right)=0 ;
$$

hence $h_{j} x_{j} \in A_{1}$ and $g\left(h_{j} x_{j}\right)=0$. Since $q x_{j} \in A_{i}, \quad q\left(h_{j} x_{j}\right)=h_{j}\left(q x_{j}\right)=0$. Thus

$$
g(x)=s_{1}\left(g x_{1}\right)+\cdots+s_{n}\left(g x_{n}\right)=s_{1}\left(q x_{1}\right)+\cdots+s_{n}\left(q x_{n}\right)=q(x) .
$$

Therefore, $g-q \in H_{i+1}$. We let $p_{i+1}=p_{i}+q$; then $f \equiv p_{i+1}\left(\bmod H_{i+1}\right)$, and we have verified the induction.

Thus we have associated with $f$ a sequence $\left\{p_{i}\right\}$ of elements of $R$. If $n \leqq m$, then $p_{n}-p_{m}=\left(p_{n}-f\right)+\left(f-p_{m}\right) \in H_{n}+H_{m}=H_{n}$. Therefore, $\left(p_{n}-p_{m}\right) A_{n}=0$, and so by Theorem 3.4 (2) $p_{n}-p_{m} \in P^{n}$. Thus $\left\{p_{i}\right\}$ is a Cauchy sequence in $R$; and since $R$ is complete, $p_{i} \rightarrow p \in R$. Since $P^{i} \subset H_{i}, p_{i} \rightarrow p$ in $H$. But $p_{i} \rightarrow f$ in $H$, and so $f=p \in R$. Thus $H=R$ and the theorem is proved.

COROLlary 3.8 .

(1) Let $y, x_{1}, \cdots, x_{n} \in E$. Then $\cap O\left(x_{j}\right) \subset O(y)$ if and only if there exist $r_{1}, \cdots, r_{n} \in R_{p}$ such that $y=r_{1} x_{1}+\cdots+r_{n} x_{n}$.

(2) Every R-homomorphism from one submodule of $E$ into another can be realized by multiplication by an element of $\bar{R}_{p}$.

Proof.

(1) This is an immediate consequence of Proposition 2.8, Theorem 
3.7, and the definition of the operation of $\bar{R}_{p}$ on $E$.

(2) Since $E$ is injective, every $R$-homomorphism from one submodule of $E$ to another can be extended to an endomorphism of $E$.

DeFinition. Let $P$ be a prime ideal of $R$, and let $P^{(i)}=$ $Q_{1} \cap \cdots \cap Q_{t} \cap Q_{t+1} \cap Q_{n}$ be an irredundant decomposition of the symbolic prime power $P^{(i)}$ by irreducible ideals $Q_{m}$ such that $Q_{m} \not \supset P^{(i-1)}$ for $m=1, \cdots, t$ and $Q_{m} \supset P^{(i-1)}$ for $m=t+1, \cdots, n$. We say that this is a minimal decomposition of $P^{(i)}$ and that $P^{(i)}$ belongs to $t$, if $t$ is the smallest integer for which we can obtain such a decomposition. Clearly for $i>0 P^{(i)}$ has a minimal decomposition; and $t>0$ if and only if $P^{(i)} \neq P^{(i-1)}$.

THEOREM 3.9. Let $P$ be a prime ideal of $R, E=E(R / P)$, and assume that $P^{(i+1)}$ belongs to $t$. Then the dimension of $A_{i+1} / A_{i}$ as a vector space over the quotient field of $R / P$ is equal to $t$.

Proof. We can assume without loss of generality that $R$ is a local ring with maximal ideal $P$. Then $P^{(i)}=P^{i}$ for every $i$. Let $F^{i+1}=$ $Q_{1} \cap \cdots \cap Q_{t} \cap Q_{t+1} \cap \cdots \subset Q_{n}$ be a minimal decomposition of $P^{i+1}$, where $Q_{m} \not \supset P^{i}$ for $m=1, \cdots, t$ and $Q_{m} \supset P^{i}$ for $m=t+1, \cdots, n$. By Lemma 3.2 (1) we can find $x_{m} \in A_{i+1}$ such that $O\left(x_{m}\right)=Q_{m}$ for $m=1, \cdots, n$. Let $\bar{x}_{m}=x_{m}+A_{i}$; we will show that $\bar{x}_{1}, \cdots, \bar{x}_{t}$ form a basis for $A_{i+1} / A_{i}$ over $R / P$.

(1) $\bar{x}_{1}, \cdots, \bar{x}_{t}$ are linearly independent over $R / P$. Suppose that $\bar{r}_{1} \bar{x}_{1}+\cdots+\bar{r}_{s} \bar{x}_{s}=0$. where $s \leqq t$ and $\bar{r}_{j}=r_{j}+P$ for some $r_{j} \in R$. We can assume that $\bar{r}_{i} \neq 0$ for $j=1, \cdots, s$. Now $r_{1} x_{1}+\cdots+r_{s} x_{s}=y \in A_{i}$; and so $r_{1} x_{1}=-r_{2} x_{2}-\cdots-r_{s} x_{s}+y$. Applying Lemma 3.2 (2) we have $O\left(x_{1}\right) \supset O\left(x_{2}\right) \cap \cdots \cap O\left(x_{s}\right) \cap O(y)$. Hence $P^{i+1}=O\left(x_{2}\right) \cap \cdots \cap O\left(x_{s}\right) \cap O(y)$. We can refine this irreducible decomposition of $P^{i+1}$ to an irredundant one ; but since $O(y) \supset P^{i}$, we will then have $t-1$, or fewer, components not containing $P^{i}$. This contradicts the minimal nature of $t$; and therefore, $\bar{x}_{1}, \cdots, \bar{x}_{t}$ are linearly independent over $R / P$.

(2) $\bar{x}_{1}, \cdots, \bar{x}_{t}$ span $A_{i+1} / A_{i}$ over $R / P$. For let $x \in A_{i+1}$; then $O(x) \supset P^{i+1}=\cap O\left(x_{m}\right)$. Therefore, by Corollary 3.7 (1) there exist $r_{1}, \cdots, r_{n} \in R$ such that $x=r_{1} x_{1}+\cdots+r_{n} x_{n}$. Since $Q_{m} \supset P^{i}$ for $m=t+1, \cdots, n$, it follows that $x_{m} \in A_{i}$ for $m=t+1, \cdots, n$. Thus $x+A_{i}$ is a linear combination of $\bar{x}_{1}, \cdots, \bar{x}_{t}$ with coefficients in $R / P$.

The main part of the following theorem was communicated to me by A. Rosenberg and D. Zelinsky.

TheOREM 3.10. With the notation of Theorem 3.9 let $K$ be the quotient fleld of $R / P$. Then $A_{i+1} / A_{i}$ is isomorphic as a vector space over $K$ to the dual space of $P^{i} / P^{i+1} \otimes_{R / P} K$. 
Proof. By Lemma 3.5 and Theorem 3.6 we can assume that $R$ is a local ring with maximal ideal $P$; and then we must prove that $A_{i+1} / A_{i}$ is isomorphic as a vector space over $R / P$ to the dual space of $P^{i} / P^{i+1}$. By [9, Lemma 1] we know that $A_{i+1} / A_{i}$ is isomorphic over $R / P$ to $\operatorname{Hom}_{R}\left(P^{i} / P^{i+1}, E\right)$. It is clear that any $R$-homomorphism of $P^{i} / P^{i+1}$ into $E$ has its range in $A_{1}$ (which is isomorphic to $R / P$ ), and is actually an $R / P$-homomorphism. Thus we can identify $\operatorname{Hom}_{R}\left(P^{i} / P^{i+1}, E\right)$ with $\operatorname{Hom}_{R \mid P}\left(P^{i} \mid P^{i+1}, R / P\right)$. Since the latter is the dual space of $P^{i} / P^{i+1}$ as a vector space over $R / P$, this concludes the proof.

Theorem 3.10 provides a second proof that $A_{i+1} / A_{i}$ is a finite dimensional vector space over $K$; and in addition shows that this dimension is equal to the dimension over $K$ of $P^{i} / P^{i+1} \otimes_{R / P} K$. Combining this result with Theorem 3.9 we see that $P^{(i+1)}$ belongs to $t$ if and only if $P^{i} / P^{i+1} \otimes_{R / P} K$ has dimension $t$ over $K$.

\section{THEOREM 3.11.}

(1) If $P$ is a maximal ideal of $R$, then $A_{i} \subset E(R / P)$ is a finitely generated $R$-module for every integer $i$; and thus $E(R / P)$ is a countably generated $R$-module.

(2) $R$ has the minimum condition on ideals if and only if every indecomposable, injective $R$-module is finitely generated.

Proof.

(1) The proof is by induction on $i$. Since the case $i=0$ is trivial, assume that $A_{i-1}$ is finitely generated. By Theorem 3.9 we can find $x_{1}, \cdots, x_{n} \in A_{i}$ such that if $x \in A_{i}$, then there exists $b \in R-P$ such that $b x$ is in the module generated by $x_{1}, \cdots, x_{n}$ and $A_{i-1}$. Since $P$ is maximal and $P x \subset A_{i-1}, x$ is in the module generated by $A_{i-1}$ and $x_{1}, \cdots, x_{n}$. Thus $A_{i}$ is finitely generated.

(2) Suppose that $R$ has the minimum condition on ideals. Let $P$ be a prime ideal of $R$; then $P$ is a maximal ideal, and there exists an integer $i$ such that $P^{i}=P^{i+1}=F^{i+2}=\cdots$. Therefore, $E(R / P)=A_{i}$; and by (1) $E(R / P)$ is finitely generated over $R$. Since $E(R / P)$ is typical, all indecomposable, injective $R$-modules are finitely generated.

Conversely, assume that every indecomposable, injective $R$-module is finitely generated. Let $P$ be a prime ideal of $R$. Then $A_{1} \subset E(R / P)$ is finitely generated over $R / P$. But $A_{1}$ is isomorphic to the quotient field of $R / P$, and hence $R / P$ is a field. Thus prime ideals of $R$ are maximal; and since $R$ is a Noetherian ring, this implies that $R$ has the minimum condition on ideals.

\section{Duality}

Definition. Let $R$ be any $\operatorname{ring}$ and $M$ an $R$-module. Then we say 
that $M$ has ACC (Ascending Chain Condition) if every ascending chain of submodules of $M$ terminates, and we say that $M$ has DCC (Descending Chain Condition) if every descending chain of submodules of $M$ terminates.

Proposition 4.1. Let $R$ be a left-Noetherian ring, and $M$ an $R$ module having DCC. Then $E(M)$ is a direct sum of a finite number of indecomposable injective $R$-modules.

Proof. By Theorem 2.5 $E(M)=\sum \oplus E_{a}(a \in A)$, where the $E_{a}$ 's are indecomposable, injective $R$-modules. Assume that the index set $A$ is infinite. Then $A$ has a properly descending chain of subsets: $A \supset A_{1} \supset A_{2} \supset \cdots$. Define the submodule $C_{i}$ of $E(M)$ by $C_{i}=\sum \oplus E_{b}\left(b \in A_{i}\right)$. Then $M \supset\left(C_{1} \cap M\right) \supset\left(C_{2} \cap M\right) \supset \cdots$ is a descending chain of submodules of $M$. Since $M \cap E_{a} \neq 0$ for all $a \in A$, the chain is properly descending. This contradiction shows that $A$ is finite.

Definition. Let $R$ be any $\operatorname{ring}$ and $M$ an $R$-module. For any positive integer $n$ let $M^{n}$ denote a direct sum of $n$ copies of $M$. If $x=\left(x_{1}, \cdots, x_{n}\right) \in M^{n}$, where $x_{1} \in M$, we will denote $x$ by $\left(x_{i}\right)$. For a fixed pair $R^{n}, M^{n}$ we make the following definitions : let $S$ be a subset of $R^{n}$ and $B$ a subset of $M^{n}$; then define $S^{\prime}=\left\{\left(x_{i}\right) \in M^{n} \mid \sum r_{i} x_{i}=0\right.$ for all $\left.\left(r_{i}\right) \in S\right\}$ and $B^{\prime}=\left\{\left(r_{i}\right) \in R^{n} \mid \sum r_{i} x_{i}=0\right.$ for all $\left.\left(x_{i}\right) \in B\right\}$. If $R$ is commutative, then $S^{\prime}$ is a submodule of $M^{n}$ and $B^{\prime}$ is a submodule of $R^{n}$.

THEOREM 4.2. Let $R$ be a commutative, Noetherian, complete, local ring, $P$ its maximal ideal, and $E=E(R / P)$. Take a fixed pair $R^{n}, E^{n}$; let $S$ be a submodule of $R^{n}$ and $B$ a submodule of $E^{n}$. Then:

(1) $S^{\prime} \cong \operatorname{Hom}_{R}\left(R^{n} / S, E\right)$, and $E^{n} / S^{\prime} \cong \operatorname{Hom}_{R}(S, E)$, and $S^{\prime \prime}=S$.

(2) $B^{\prime} \cong \operatorname{Hom}_{R}\left(E^{n} / B, E\right)$, and $R^{n} / B^{\prime} \cong \operatorname{Hom}_{R}(B, E)$, and $B^{\prime \prime}=B$.

Thus if $A$ is a submodule or factor module of either $R^{n}$ or $E^{n}$, then $\operatorname{Hom}_{R}\left(\operatorname{Hom}_{R}(A, E), E\right) \cong A$. The operation' is a lattice anti-isomorphism of the submodules of $R^{n}$ and $E^{n}$. Therefore, $E^{n}$ has DCC.

Proof.

(1) $R^{n} / S$ is generated by elements $u_{i}, i=1, \cdots, n$, such that $\left(r_{i}\right) \in S \Leftrightarrow \sum r_{i} u_{i}=0$. Let $f \in \operatorname{Hom}_{R}\left(R^{n} / S, E\right)$ and define $\phi: \operatorname{Hom}_{R}\left(R^{n} / S, E\right) \rightarrow S^{\prime}$ by $\phi(f)=\left(f u_{i}\right)$. Clearly $\phi$ is a well-defined $R$-homomorphism. If $\phi(f)=0$, then $f u_{i}=0$ for all $i$, and so $f=0$. Thus $\phi$ is one-to-one. If $\left(x_{i}\right) \in S^{\prime}$, define $f: R^{n} / S \rightarrow E$ by $f u_{i}=x_{i}$; and then $\phi(f)=\left(x_{i}\right)$. Thus $\phi$ is onto, and so $\operatorname{Hom}_{R}\left(R^{n} / S, E\right) \cong S^{\prime}$.

From the exact sequence :

$$
0 \rightarrow S \rightarrow R^{n} \rightarrow R^{n} / S \rightarrow 0
$$


we derive the commutative diagram :

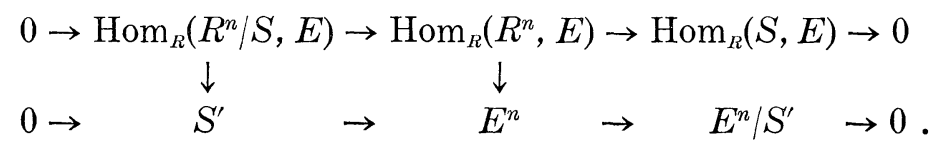

Since $E$ is injective, the top row is exact. Of course the bottom row is exact, and the vertical maps are isomorphisms. Thus $\operatorname{Hom}_{R}(S, E) \cong$ $E^{n} / S^{\prime}$.

Clearly $S \subset S^{\prime \prime} \subset R^{n}$. Suppose that $\left(s_{i}\right) \in S^{\prime \prime}$, but $\left(s_{i}\right) \notin S$. Then $y=\sum s_{i} u_{i} \neq 0 \in R^{n} / S$. Now $O(y) \subset P$, and so by Proposition 2.8 there exists an $R$-homomorphism $g: R^{n} / S \rightarrow E$ such that $g(y) \neq 0$. However, $g(y)=\sum s_{i} g\left(u_{i}\right)=0$, since $\left(g u_{i}\right) \in S^{\prime}$. This is a contradiction, and so $S=S^{\prime \prime}$.

(2) Let $\left(r_{i}\right) \in B^{\prime}$ and $\left(y^{\prime}\right) \in E^{n}$. Define $\Phi: B^{\prime} \rightarrow \operatorname{Hom}_{R}\left(E^{n} / B, E\right)$ by $\Phi\left(r_{i}\right)\left[\left(y_{i}\right)+B\right]=\sum r_{i} y_{i}$. Then $\Phi$ is a well defined $R$-homomorphism. If $\Phi\left(r_{i}\right)=0$, then $\sum r_{i} y_{i}=0$ for all $\left(y_{i}\right) \in E^{n}$. Thus $r_{i} E=0$ for all $i$. Since $E$ is a faithful $R$-module by Theorem 3.7. $r_{i}=0$ for all $i$. Thus $\Phi$ is one-to-one. Let $f \in \operatorname{Hom}_{R}\left(E^{n} / B, E\right)$, and for each $i=1, \cdots, n$ let $E_{i}$ be the $i$ th component of $E^{n}$. Then the composite mapping : $E \rightarrow E_{i} \rightarrow$ $E^{n} \rightarrow E^{n} / B^{f} \rightarrow E$ induces an $R$-endomorphism of $E$, and so by Theorem 3.7 can be realized by multiplication by an element $r_{i} \in R$. Thus if $\left(y_{i}\right) \in E^{n}, f\left[\left(y_{i}\right)+B\right]=\sum r_{i} y_{i}$. If $\left(x_{i}\right) \in B$, then $\sum r_{i} x_{i}=f\left[\left(x_{i}\right)+B\right]=0$; and thus $\left(r_{i}\right) \in B^{\prime}$. Clearly $\Phi\left(r_{i}\right)=f$, and thus $\Phi$ is onto. Therefore, $B^{\prime} \cong \operatorname{Hom}_{R}\left(E^{n} / B, E\right)$.

From the exact sequence :

$$
0 \rightarrow B \rightarrow E^{n} \rightarrow E^{n} / B \rightarrow 0
$$

we derive the commutative diagram:

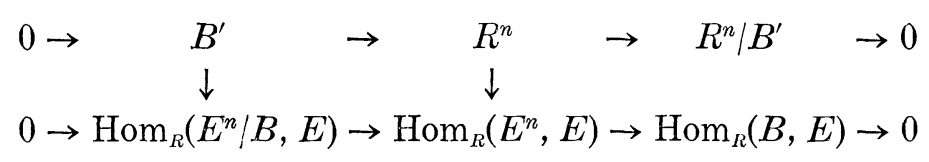

The top row is exact, the vertical maps are isomorphisms; and since $E$ is injective, the bottom row is exact. Thus $R^{n} / B^{\prime} \cong \operatorname{Hom}_{R}(B, E)$.

Clearly $B \subset B^{\prime \prime} \subset E^{n}$. Suppose that $\left(z_{i}\right) \in B^{\prime \prime}$, but $\left(z_{i}\right) \notin B$. Since $O\left(\left(z_{i}\right)+B\right) \subset P$, we can find by Proposition 2.8 an $R$-homomorphism $f: E^{n} / B \rightarrow E$ such that $f((z)+B) \neq 0$. However, $f=\Phi\left(r_{i}\right)$, where $\left(r_{i}\right) \in B^{\prime}$; and thus $f\left(\left(z_{i}\right)+B\right)=\bar{\varphi}\left(r_{i}\right)((z)+B)=\sum r_{i} z_{i}=0$, since $\left(z_{i}\right) \in B^{\prime \prime}$. This contradiction shows that $B=B^{\prime \prime}$.

\section{REMARKS.}

(1) It follows easily from Proposition 2.8 that if $R$ is any ring, $C$ an injective $R$-module which contains a copy of every simple 
$R$-module, and $M$ any $R$-module, then $M$ is imbedded in a natural way in $\operatorname{Hom}_{R}\left(\operatorname{Hom}_{R}(M, C), C\right)$. G. Azumaya has also obtained this result [2, Prop. 6]. However, his other duality statements concern non-commutative rings with minimum conditions on both left and right ideals.

(2) It is easily seen that if $R$ is a commutative, Noetherian ring, and $C$ an injective $R$-module such $\operatorname{Hom}_{R}\left(\operatorname{Hom}_{R}(A, C), C\right) \cong A$ for every cyclic $R$-module $A$, then $R$ is a finite direct sum of complete, local rings $R_{i}$. And if $C_{i}$ is the $R_{i}$-component of $C$, and $P_{i}$ the maximal ideal of $R_{i}$, then $C_{i} \cong E\left(R_{i} / P_{i}\right)$.

Corollary 4.3. Let $R$ be a commutative, Noetherian, complete, local ring ; $P$ its maximal ideal ; and $E=E(R / P)$. Then:

(1) An $R$-module $M$ has ACC if and only if it is a homomorphic image of $R^{n}$ for some $n$.

(2) An R-module $M$ has DCC if and only if it is a submodule of $E^{n}$ for some $n$.

(3) If $X, Y$ are the categories of $R$-modules with ACC and DCC, respectively, then the contravariant, exact functor $\operatorname{Hom}_{R}(, E)$ establishes $a$ one-to-one correspondence $X \leftrightarrow Y$. In particular, $\operatorname{Hom}_{R}\left(\operatorname{Hom}_{R}(M, E), E \cong M\right.$ for $M$ in either category.

Proof.

(1) This is true for any left-Noetherian ring.

(2) By Theorem 4.2 every submodule of $E^{n}$ has DCC. On the other hand let $M$ be a module with DCC. By Proposition 4.1 $E(M)=$ $C_{1} \oplus \cdots \oplus C_{n}$, where the $C_{i}$ 's are indecomposable, injective $R$-modules. Let $x \neq 0 \in M$. Then there exists an integer $j$ such that $P^{j} x=P^{j+1} x$, and so $P^{j} \subset O(x)+P^{j+1}$. This implies that $P^{j} \subset O(x)$ [8, Prop. 4.2.1]; and thus $O(x)$ is a $P$-primary ideal. It follows from Lemma $3.2(1)$ that $C_{i} \cong E$ for $i=1, \cdots, n$.

(3) This follows immediately from (1), (2), and Theorem 4.2.

\section{REFERENCES}

1. G. Azumaya, Corrections and supplementaries to my paper concerning Krull-RemakSchmidi's theorem, Nagoya Maih. 1 (1950), 117-124.

2. - - A duality theory for injective modules, (forthcoming).

3. H. Cartan and S. Eilenberg, Homological algebra, Princeton University Press, 1956.

4. B. Eckmann and A. Schopf, Über injective Moduln, Arch. Math., 4 (1953), 75-78.

5. I. Kaplansky, Infinite Abelian groups, University of Michigan Press, 1954.

6. Y. Kawada, K. Morita, and H. Tachikawa, On injective modules, Math. Z. 68, (1957), 216-226.

7. H. Nagao and T. Nakayama, On the structure of $\left(\boldsymbol{M}_{0}\right)$ and $\left(\boldsymbol{M}_{u}\right)$-modules, Math. $Z$. 59 (1953), 164-170.

8. D. Northcott, Ideal theory, Cambridge University Press, 1953.

9. A. Rosenberg and D. Zelinsky, Finiteness of the injective hull, (forthcoming). 


\section{PACIFIC JOURNAL OF MATHEMATICS}

\section{EDITORS}

David Gilbarg

Stanford University

Stanford, California

R. A. Beaumont

University of Washington

Seattle 5, Washington

\author{
A. L. Whiteman
}

University of Southern California

Los Angeles 7, California

E. G. Straus

University of California

Los Angeles 24, California

\section{ASSOCIATE EDITORS}

\author{
E. F. BECKENBACH \\ C. E. BURGESS \\ M. HALL \\ E. HEWITT
}

\author{
A. HORN \\ V. GANAPATHY IYER \\ R. D. JAMES \\ M. S. KNEBELMAN
}

L. NACHBIN
I. NIVEN
T. G. OSTROM
H. L. ROYDEN

M. M. SCHIFFER

G. SZEKERES

F. WOLF

K. YOSIDA

\section{SUPPORTING INSTITUTIONS}

\author{
UNIVERSITY OF BRITISH COLUMBIA \\ CALIFORNIA INSTITUTE OF TECHNOLOGY \\ UNIVERSITY OF CALIFORNIA \\ MONTANA STATE UNIVERSITY \\ UNIVERSITY OF NEVADA \\ OREGON STATE COLLEGE \\ UNIVERSITY OF OREGON \\ UNIVERSITY OF SOUTHERN CALIFORNIA
}

\author{
STANFORD UNIVERSITY \\ UNIVERSITY OF UTAH \\ WASHINGTON STATE COLLEGE \\ UNIVERSITY OF WASHINGTON \\ AMERICAN MATHEMATICAL SOCIETY \\ CALIFORNIA RESEARCH CORPORATION \\ HUGHES AIRCRAFT COMPANY \\ THE RAMO-WOOLDRIDGE CORPORATION
}

Mathematical papers intended for publication in the Pacific Journal of Mathematics should be typewritten (double spaced), and the author should keep a complete copy. Manuscripts may be sent to any of the editors. All other communications to the editors should be addressed to the managing editor, E. G. Straus at the University of California, Los Angeles 24, California.

50 reprints per author of each article are furnished free of charge; additional copies may be obtained at cost in multiples of 50 .

The Pacific Journal of Mathematics is published quarterly, in March, June, September, and December. The price per volume (4 numbers) is $\$ 12.00$; single issues, $\$ 3.50$. Back numbers are available. Special price to individual faculty members of supporting institutions and to individual members of the American Mathematical Society: $\$ 4.00$ per volume; single issues, $\$ 1.25$.

Subscriptions, orders for back numbers, and changes of address should be sent to Pacific Journal of Mathematics, 2120 Oxford Street, Berkeley 4, California.

Printed at Kokusai Bunken Insatsusha (International Academic Printing Co., Ltd.), No. 6, 2-chome, Fujimi-cho, Chiyoda-ku, Tokyo, Japan.

\section{PUBLISHED BY PACIFIC JOURNAL OF MATHEMATICS, A NON-PROFIT CORPORATION}

The Supporting Institutions listed above contribute to the cost of publication of this Journal, but they are not owners or publishers and have no responsibility for its content or policies. 


\section{Pacific Journal of Mathematics}

\section{Vol. 8, No. 3 \\ May, 1958}

Michael Israel Aissen, A set function defined for convex plane domaines... . 383

Robert Ellis, Distal transformation groups ................... 401

Ciprian Foias, On a commutative extension of a commutative Banach algebra ....................................... 407

Jerry William Gaddum, Linear inequalities and quadratic forms ......... 411

Allen A. Goldstein and Elliott Ward Cheney, Jr., A finite algorithm for the solution of consistent linear equations and inequalities and for the Tchebycheff approximation of inconsistent linear equations...........

William L. Hart and T. S. Motzkin, Proof of the fundamental theorem on implicit functions by use of composite gradient corrections .......... 429

Henry Berge Helson, Conjugate series and a theorem of Paley .......... 437

Wu-Chung Hsiang, Abelian groups characterized by their independent subsets....................................... 447

John W. Lamperti, On the isometries of certain function-spaces ........ 459

Karel DeLeeuw and Walter Rudin, Extreme points and extremum problems

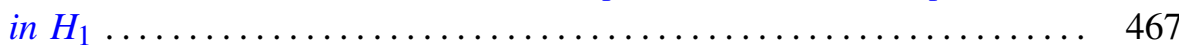

Eugene Lukacs, Some extensions of a theorem of Marcinkiewicz........ 487

George W. Mackey, Multiplicity free representations of finite groups ..... 503

Eben Matlis, Injective modules over Noetherian rings ............. 511

John William Neuberger, Continuous products and nonlinear integral equations

Lawrence Edward Payne and Hans F. Weinberger, New bounds for solutions of second order elliptic partial differential equations...

William T. Reid, A Prüfer transformation for differential systems ........ 575

Howard L. Rolf, The free lattice generated by a set of chains ...

K. M. Saksena, Inversion and representation theorems for a generalized

Laplace integral....................................... 597

Daniel Shanks, Two theorems of Gauss......................... 609

Paul Slepian, On the Lebesgue area of a doubled map ............... 613

Otto Szász and Nelson Paul Yeardley, Jr., The representation of an analytic function by general Laguerre series . ..................... 621

Alan C. Woods, On two-dimensional convex bodies ................. 635 